INRA Prod. Anim., 2017, 30 (3), 241-254

\title{
Les services rendus par les systèmes de production de foie gras agroforestiers : l'exemple de l'association entre oies et noyers en Périgord
}

\author{
M. BIJJA', J. ARROYO', F. LAVIGNE', J.-P DUBOIS ${ }^{l}$, L. FORTUN-LAMOTHE \\ ${ }^{1}$ ASSELDOR-Station d'expérimentation appliquée et de démonstration sur l'oie, \\ La Tour de Glane, 24420, Coulaures, France \\ ${ }^{2}$ GenPhySE, Université de Toulouse, INRA, INPT, ENVT, 31320, Castanet-Tolosan, France \\ Courriel : laurence.lamothe@inra.fr
}

L'élevage est aujourd'hui l'objet de nombreuses critiques et pourtant il a été montré qu'il rend de nombreux services aux territoires et à la société. Le foie gras n'est pas épargné et fait l'objet de débats contradictoires : inscrit au patrimoine culturel et gastronomique français, sa production est aussi largement questionnée sur la pratique du gavage. L'agroforesterie est un ensemble de pratiques qui permet d'élargir les bénéfices rendus par l'élevage. Cet article examine l'ensemble des services rendus par les systèmes de production de foie gras agroforestiers.

L'élevage est souvent pointé du doigt pour ses impacts environnementaux : pollutions de l'eau et des sols, émissions de gaz à effet de serre, utilisation de la ressource en eau et compétition pour l'usage des terres (Steinfeld et al 2006, Gerber et al 2013). C'est dans ce contexte, que de nombreux courants de pensée se développent pour maîtriser les impacts environnementaux de l'élevage tout en préservant leur capacité de production : élevage de précision, économie circulaire, agriculture écologiquement intensive ou agroécologie. Cette dernière cherche à refonder les systèmes agricoles en mobilisant les concepts de l'écologie. Son application aux systèmes d'élevage passe notamment par un couplage des productions animales et végétales au sein des systèmes de production et/ou des territoires afin d'augmenter la diversité, de limiter l'utilisation des intrants et les problèmes de pollutions liés aux déjections animales (Dumont et al 2013). L'agroforesterie, définie comme l'association d'arbres à des cultures végétales et/ou à de l'élevage sur une même parcelle («International Center for Research in Agroforestry ", ICRAF, http://www.ciesin.org/IC/icraf/ICRAF.h $\underline{\text { tml) }}$ et dont les interactions écologiques et/ou économiques entre les composantes arborées et non arborées sont significatives (Nair 1993), est un exemple particulier de ce couplage. Ces associations ne sont pas nouvelles et des systèmes comme les prés-vergers en Normandie et dans les Pays de la Loire qui produisent du cidre et de la viande de mouton ou encore l'association de chênes lièges et de troupeaux porcins et bovins en Espagne et au Portugal sont des exemples de systèmes agroforestiers traditionnels qui sont toujours d'actualité.

L'association entre des oies élevées pour la production de foie gras et des noyers qui produisent à la fois des fruits et du bois d'œuvre est également une tradition séculaire dans le Périgord. Ses intérêts fondateurs, surtout pratique et économique, reposent sur le fait que les arbres apportent de l'ombre aux animaux tandis que les déjections des volailles fertilisent les arbres. C'est la raison pour laquelle l'aménagement agroforestier des parcours de volailles fait aujourd'hui l'objet d'un intérêt croissant. On peut ainsi noter la création en 2015 en Périgord, du Groupement d'Intérêt Économique et Environnemental (GIEE) Agroforesterie porté par l'association foie gras du Périgord (http://agriculture. gouv.fr/sites/minagri/files/agroforesterie_plantation_darbres04112015.pdf) avec comme partenaires engagés des groupements de producteurs, l'Etablissement public local d'enseignement et de formation professionnelle agricoles du Périgord et la Ferme d'Expérimentation et de démonstration de l'Oie et du Canard (FEOC). Ces initiatives sont encouragées par les politiques publiques et les évolutions réglementaires et fiscales (circulaire GGPAAT/SDBE/SDBF/ C2010-3035 du 6 avril 2010 du Ministère de l'Alimentation, de l'Agriculture et de la Pêche). Il semble donc intéressant de qualifier plus largement l'ensemble des services rendus par ces systèmes d'élevage particuliers comme cela a été fait récemment pour les autres filières d'élevage en Europe (Dumont et al 2016).

Cet article propose de qualifier la diversité des services rendus par les systèmes de production de foie gras agroforestiers en prenant l'exemple de l'association oies/noyers en Périgord.

\section{1 / Méthodologie}

\section{1 / Le modèle d'étude : l'asso-} ciation oies/noyers en Périgord

a) Le territoire : le Périgord

Le Périgord est un ancien comté qui recouvrait l'actuel département français de la Dordogne, dans la région NouvelleAquitaine. Ce territoire de $9000 \mathrm{~km}^{2}$, qui aujourd'hui s'étend au-delà de la Dordogne en incluant une partie des départements du Lot, de la Corrèze et de la Charente, est essentiellement rural et marqué par un important patrimoine historique mais est également célèbre pour sa gastronomie. On note en premier lieu le foie gras et les confits de volailles, mais également la truffe noire, les noix, les cèpes ou les châtaignes. 


\section{b) Le foie gras}

\section{L'oie dans la production de foie gras}

Le foie gras est produit à partir de deux espèces de palmipèdes : l'oie (Anser anser) ou le canard, que ce soit le canard de barbarie (Cairina moschata) ou le canard mulard (Cairina moschata $\times$ Anas platyrhynchos ; Baéza et al 2013). La production de foie gras de canard domine le marché du foie gras aussi bien au niveau mondial qu'en France, et la production de foie gras d'oie est aujourd'hui très minoritaire $(<10 \%$ du tonnage mondial et $<5 \%$ du tonnage français). Elle est présente dans de nombreuses régions françaises mais est très majoritairement localisée dans le sud-ouest (NouvelleAquitaine et Occitanie : $80 \%$ du cheptel), notamment dans le Périgord (CIFOG, 2015).

Ce système de production est intéressant pour l'étude de la diversité des services rendus car l'élevage regroupe généralement sur la ferme les phases de croissance, de préparation au gavage, de gavage et de transformation avec une commercialisation qui se fait le plus souvent en circuit courts c'est-à-dire sans intermédiaire, directement du producteur au consommateur.

Le système de production de foie gras d'oie en Périgord

Il existe environ 70 producteurs de foie gras d'oie en Périgord. Le système d'élevage des oies pour la production de foie gras a été décrit par Arroyo et al $(2012 \mathrm{c})$. On peut rappeler brièvement que celui-ci comporte quatre étapes principales : la phase de démarrage (de 1 à 40 jours d'âge environ), de croissance (de 40 à 80-100 jours d'âge environ), de préparation au gavage (de 80-100 à 100-120 jours d'âge environ) et de gavage (de 100-120 à 120-140 jours d'âge environ).

\section{c) La production de noix en Périgord}

La France est le neuvième pays producteur de noix dans le monde et le $2^{\text {ème }}$ au niveau européen (36 000 t/an ; $1 \%$ de la production mondiale). Le Périgord contient 9000 ha de noyers. En effet, les sols argilo-calcaires du jurassique et du crétacé qui composent $90 \%$ de ce terroir sont propices à la culture du noyer. De plus, le climat avec une température moyenne annuelle supérieure à $10,5^{\circ} \mathrm{C}$ et une pluviométrie moyenne annuelle supérieure à $750 \mathrm{~mm}$, optimise la qualité des fruits. Enfin, le relief vallonné de cette région permet de protéger les noyers du gel, auquel il est sensible, en étant soit abrité dans des vallées, soit exposé sur des coteaux à moins de $500 \mathrm{~m}$ d'altitude.

\section{d) L'association oies/noyers}

L'association entre la production de foie gras d'oie et de noix est d'origine séculaire et la grande majorité des producteurs de foie gras qui se trouvent dans la zone d'Appellation d'origine Contrôlée (AOC) Noix du Périgord, pratiquent cette association. Ce système d'élevage est emblématique d'un système agroforestier basé sur des productions animales et végétales ancestrales dans ce territoire. En effet, en Périgord, quasiment tous les producteurs qui possèdent des surfaces pour l'élevage des oies y ont implanté du noyer. La terre est appropriée à cette essence arborescente et la valorisation commerciale des noix est portée par la dynamique territoriale (voir § 4.2.). C'est pourquoi, il nous a semblé un bon modèle pour étudier l'intérêt de l'agroforesterie sur la diversité des services rendus par l'élevage.

\section{2. / L'évaluation des services rendus par l'élevage}

La notion de service recouvre des réalités différentes suivant les disciplines. Dans ce travail, nous avons adapté le cadre conceptuel proposé par Ryschawy et al (2015) qui distingue quatre catégories de services : les services d'approvisionnement (la production matérielle et énergétique des écosystèmes), de qualité environnementale, de patrimoine et qualité de vie et de vitalité territoriale, auquel nous avons ajouté une catégorie sur les externalités négatives (ou disservices).

Pour réaliser l'évaluation des services rendus par les systèmes d'élevage agroforestiers de foie gras nous avons combiné 1) des données expérimentales, notamment pour caractériser l'intérêt de l'association oies/noyers sur les réponses biologiques (croissance des arbres ou production de fruit, comportement des animaux, biodiversité, disservices...) ou pédologiques (fertilité des sols) et 2) des données de la littérature, pour les autres dimensions.

Les données expérimentales sont issues d'expérimentations réalisées à la FEOC (Domaine de Coulaures, La tour de Glane, 24420 COULAURES). Elle a été créée en 1992 par l'association des éleveurs de Dordogne (ASSELDOR) et est aujourd'hui une des quatre entités impliquées dans le continuum recherche-développementtransfert pour la filière foie gras avec l'INRA (Institut National de la Recherche Agronomique), l'ITAVI (InstitutTechnique de l'AVIculture) et le CEPSO (Centre d'Études des Palmipèdes du Sud-Ouest Organisme Inter Établissement du Réseau des Chambres d'Agriculture d'Aquitaine et Midi-Pyrénées). Ces quatre entités sont regroupées au sein du Groupement
d'Intérêt Scientifique (GIS) Palmipôle (http://www.cepso.chambagri.fr/le-palmipole.html) depuis 2014.

La FEOC comporte 4 bâtiments d'élevage adaptés à l'expérimentation avec accès à des parcours extérieurs herbagés ou agroforestiers (pour un total de 2950 oies), ainsi qu'une salle de gavage (pour 300 oies). Le parcours agroforestier, d'une surface d'un hectare, a été planté de 75 noyers en 1998 puis de 65 noyers en 2002 ( 8 mètres d'espace entre les noyers d'un même rang et $8 \mathrm{~m}$ entre les rangs de noyers soit 156 arbres/ha). Sur les inter-rangs de noyers, des bandes de graminées sont implantées pour le pâturage des palmipèdes. Le verger-parcours est divisé en trois parties : une zone sans oie (témoin de 0,14 ha), et deux zones avec présence d'oies de 0,4 ha chacune. Ces parcelles ne reçoivent aucun engrais chimique. Les mesures (croissances des arbres, rendements fruitiers, analyses agronomiques, analyses sanitaires des fruits) ont été réalisées sur un parcours fréquenté par des oies adultes (600 oies reproductrices) pendant 4 années consécutives (référencées années $n$ à $n+3$ dans les tableaux) lorsque les noyers étaient âgés de 7 à 11 ans.

\section{2 / Les services d'approvision- nement}

\section{1 / Les produits animaux}

\section{a) La production de foie gras d'oie}

La production française de foie gras d'oie est de 462 tonnes (tableau 1 ; CIFOG 2015). Cela ne représente qu'une faible part de la production de foie gras en France (19 310 tonnes/an) qui est dominé par le foie gras de canard. La production de foie gras d'oie dans le Périgord peut être estimée à environ 350 tonnes avec un nombre d'oies présentes dans le Périgord estimé à 130000.

\section{b) La production de viande de palmipèdes gras}

Une oie en fin de gavage pèse 7 à $8 \mathrm{~kg}$ ce qui représente un poids de carcasse de 3,5 à 4,5 kg. Les carcasses des palmipèdes gras sont en général découpées avant la commercialisation. Chez l'oie, la valorisation se fait essentiellement sous forme de produits cuisinés. Ainsi, les cuisses-pilons, les manchons et les gésiers sont majoritairement commercialisés sous forme de confits ou dans des préparations culinaires (cassoulet). Le reste de la carcasse est utilisé pour la préparation de rillettes ou de plat élaborés (cous farcis).

En 2010, la production française totale de produits carnés issus de palmipèdes 
Tableau 1. La contribution de la filière foie gras française au service d'approvisionnement en produits animaux (d'après le rapport économique CIFOG 2015).

\begin{tabular}{|c|c|}
\hline Produit animal & $\begin{array}{c}\text { Production française } \\
\text { (en tonnes) }\end{array}$ \\
\hline Foie Gras & 19310 \\
Dont foie Gras de canard & 18848 \\
Dont foie Gras d'oie & 462 \\
\hline Viande (canards et oies) & 26000 \\
Magrets frais & 1600 \\
Magrets séchés, salés, fumés & 13000 \\
Confits & 1600 \\
Rillettes & 1000 \\
Gésiers & 1200 \\
Graisses & 55000 \\
\hline Plumes & \\
\hline
\end{tabular}

gras était d'environ $44000 \mathrm{t}$ (tableau 1 ; CIFOG, 2015). La production française de viande issue d'oies grasses était d'environ 720 tonnes sur un total de 2540 tonnes de viande d'oie (CIFOG, 2012).

\section{2 / Les produits végétaux}

a) Effet de la présence d'animaux sur la production de bois

Les noyers (Juglans regia L.) sont des arbres fournissant du bois d'œuvre qui est recherché notamment par les ébénistes, les armuriers, les menuisiers et les tourneurs. Les résultats montrent que la présence d'animaux sur un parcours arboré augmente la vitesse de croissance des arbres. Ainsi, Bijja et al (2010) ont montré que la circonférence du tronc des noyers est plus élevée dans les zones fréquentées par les oies $(+10 \%$ en moyenne sur les années $n$ à $n+3$ ) que dans la zone non fréquentée par les oies (tableau 2).

\section{b) Effet de la présence d'animaux sur la production de fruits}

Dans les noix, ce sont principalement les amandes, appelées cerneaux, qui sont consommées entières, soit fraîches soit en tant que fruit sec. Elles peuvent également être pressées pour produire l'huile de noix, très recherchée pour son goût. Cette huile possède un contenu particulièrement élevé en acides gras polyinsaturés (plus de $70 \%$ de ses lipides totaux), dont près du cinquième est sous forme d'acide alpha-linolénique, un acide gras oméga-3 essentiel. Le rendement moyen des noyers varie entre 1,5 à $3,5 \mathrm{t} / \mathrm{ha}$ (pour $100 \mathrm{arbres} / \mathrm{ha}$ ) en fonction de de la pluviométrie en l'absence d'irrigation, des amendements et des pathologies communes (bactérioses, mouche du brou).

L'effet de la présence des animaux sur la production des fruits est encore plus marqué que sur la croissance des arbres. Ainsi, la production de noix est plus élevée dans les zones fréquentées par les oies que dans la zone sans oies $(+57 \%$ en moyenne ; tableau 3). Cet effet est plus marqué dans la zone plus fréquentée $(+74 \%)$ que dans la zone peu fréquentée $(+41 \%)$ et dans l'année $n+3$ $(+98 \%)$ que dans l'année $n(+31 \%)$. Ces résultats sont d'autant plus intéressants que les données rapportées correspondant à une noyeraie âgée de 10 à 15 ans, et que cette essence arrive à son niveau de pleine production vers 11 ans d'âge. Ainsi, les productions de noix par les noyers bénéficiant de la présence des oies est $15 \%$ plus élevée que la production de référence pour une noyeraie de même configuration et de même âge mais conduite sans la présence d'animaux (2 537 vs $2200 \mathrm{~kg}$.ha-1 de noix sèche ; Prunet 2003).

\section{3 / Les coproduits}

\section{a) Les plumes et duvets}

Le corps des volailles est recouvert de différents types de plumes. A l'extérieur, se trouvent les plumes tectrices, qui protègent les animaux des intempéries. Sous les plumes tectrices, se trouvent les plumules, aussi appelées duvet, qui sont des plumes souples placées à même le corps et qui forment une couche d'isolation. Les plumes et les duvets issus des palmipèdes gras sont utilisés pour leurs qualités isolantes aussi bien dans l'industrie textile que celle de la construction. Le duvet des oies, en particulier, est utilisé pour confectionner des couettes très prisées pour leur confort et leur qualité thermique. Ces matériaux sont intéressants car leur pouvoir isolant et leur aptitude à capter l'humidité sont nettement supérieurs à ceux de tous les matériaux synthétiques. Pour faire un kilo de plume, il faut 3 à 5 oies, 6 à 7 canards mulards, 8 à 10 canards Barbarie ou 12 à 14 canards Pékins. En France, la production totale annuelle de plumes issus de palmipèdes gras est d'environ 55000 tonnes (CIFOG, 2015) et la production de plumes issus des oies grasse peut donc être estimée à environ $2700 \mathrm{t}$ /an.

\section{b) Le brou de noix}

La chair qui entoure le noyau est utilisée en décoction pour recueillir un pigment naturel. Cette teinture, brun foncé, est utilisée en menuiserie pour teinter les bois. Le brou de noix peut aussi se mordancer à l'alun afin d'être rendu insoluble et pouvoir servir pour la teinture des laines et des soies, principalement pour rabattre les teintes vives ou servir de laque.

Tableau 2. Effets de la présence des oies sur la croissance des noyers (d'après Bijja et al 2010). Moyenne \pm écart-type.

\begin{tabular}{|l|c|c|c|c|}
\hline $\begin{array}{l}\text { Circonférences } \\
\text { du tronc }(\mathbf{c m})\end{array}$ & $\begin{array}{c}\text { Zone témoin, } \\
\text { sans oie }\end{array}$ & $\begin{array}{c}\text { Zone peu fréquentée } \\
\text { par les oies }\end{array}$ & $\begin{array}{c}\text { Zone très fréquentée } \\
\text { par les oies }\end{array}$ & $\mathbf{P}>\mathbf{f}$ \\
\hline Année $\mathrm{n}$ & $38 \pm 4^{\mathrm{a}}$ & $41 \pm 4^{\mathrm{a}}$ & $42 \pm 3^{\mathrm{a}}$ & $\mathrm{NS}$ \\
Année $\mathrm{n}+1$ & $44 \pm 5^{\mathrm{b}}$ & $48 \pm 6^{\mathrm{a}}$ & $49 \pm 4^{\mathrm{a}}$ & $*$ \\
Année $\mathrm{n}+2$ & $48 \pm 5^{\mathrm{b}}$ & $52 \pm 5^{\mathrm{ab}}$ & $54 \pm 5^{\mathrm{a}}$ & $*$ \\
Année $\mathrm{n}+3$ & $51 \pm 5^{\mathrm{b}}$ & $55 \pm 4^{\mathrm{ab}}$ & $57 \pm 5^{\mathrm{a}}$ & $*$ \\
\hline
\end{tabular}

NS : $P>0,05 ;{ }^{*}: P<0,05$. Sur une même ligne, les moyennes affectées d'une même lettre ne diffèrent significativement pas entre elles. 
Tableau 3. Effets de la présence des oies sur la production de noix (d'après Bijja et al 2010). Moyenne \pm écart-type.

\begin{tabular}{|l|c|c|c|c|}
\hline $\begin{array}{c}\text { Production de noix } \\
\text { (kg/noyer) }\end{array}$ & $\begin{array}{c}\text { Zone témoin, } \\
\text { sans oie }\end{array}$ & $\begin{array}{c}\text { Zone peu fréquentée } \\
\text { par les oies }\end{array}$ & $\begin{array}{c}\text { Zone très fréquentée } \\
\text { par les oies }\end{array}$ & $\mathbf{P}>\mathbf{f}$ \\
\hline Année $\mathrm{n}$ & $5,9 \pm 2,2^{\mathrm{b}}$ & $7,1 \pm 2,1^{\mathrm{a}}$ & $8,4 \pm 2,1^{\mathrm{a}}$ & $* *$ \\
Année $\mathrm{n}+1$ & $6,8 \pm 2,8^{\mathrm{b}}$ & $7,0 \pm 2,7^{\mathrm{b}}$ & $9,6 \pm 2,7^{\mathrm{a}}$ & $* *$ \\
Année $\mathrm{n}+2$ & $7,8 \pm 4,1^{\mathrm{b}}$ & $11,1 \pm 4,0^{\mathrm{b}}$ & $16,6 \pm 4,0^{\mathrm{a}}$ & $* * *$ \\
Année $\mathrm{n}+3$ & $13,3 \pm 1,0^{\mathrm{b}}$ & $26,3 \pm 1,0^{\mathrm{a}}$ & $26,4 \pm 1,0^{\mathrm{a}}$ & $* * *$ \\
\hline
\end{tabular}

${ }^{* *}: P<0,01 ;{ }^{* *}: P<0,001$. Sur une même ligne, les moyennes affectées d'une même lettre ne diffèrent significativement pas entre elles.

\section{c) Les coques de noix}

Le décorticage des noix produit des coques. Le rapport entre poids cerneaux/ poids noix en coque est en moyenne de $42 \%$. Ainsi, si le rendement des noyers est de 1,5 à $3,5 \mathrm{t} /$ ha alors la production de coques de noix est de 0,6 à $1,5 \mathrm{t} / \mathrm{ha}$. Nous n'avons que peu d'informations quantitatives sur l'utilisation de ce coproduit mais celui-ci peut être utilisé soit comme source d'énergie (chaudière à bois), soit pour éviter le dessèchement des haies arbustives (paillage-bois) ou encore pour l'enrichissement des sols (équivalent du bois raméal fragmenté).

\section{d) La graisse d'oie}

La graisse d'oie contient 99,6\% de lipides, comme les huiles végétales. Pour un produit d'origine animale, elle est remarquablement riche en acides gras insaturés : $57 \%$ sont des acides gras monoinsaturés, $11 \%$ des polyinsaturés et $27 \%$ seulement des acides gras saturés. De ce point de vue, sa composition est assez proche de celle de l'huile d'olive, ce qui en fait un corps gras à part dans les produits issus d'animaux. Cette graisse est surtout utilisée pour la cuisine et notamment la réalisation des confits. Mais, dans certaines exploitations, elle est également utilisée pour générer de l'énergie qui sert à chauffer les bâtiments de la ferme ou à chauffer l'eau pour l'atelier de transformation avec un pouvoir calorifique de $36 \mathrm{MJ} / \mathrm{kg}$ alors celui du fioul est de $41 \mathrm{MJ} / \mathrm{kg}$ (Bliefert et al 2001).

\section{3 / Les services de préservation de la qualité environnementale}

\section{1 / Valorisation des coproduits industriels et compétition avec l'alimentation humaine}

L'élevage est une voie importante de valorisation des coproduits issus des végétaux, évitant ainsi que ceux-ci ne deviennent des déchets. Le taux d'incorporation des coproduits dans les aliments complets distribués aux oies pendant leur phase d'élevage varie généralement entre 20 et $30 \%$ pour un taux moyen de $27 \%$
(Arroyo et al 2012a, b, 2013a, b, 2015). Les besoins nutritionnels des oies reproductrices sont différents des oies en croissance (Sauveur et al 1988) et le taux moyen d'incorporation des coproduits dans leur alimentation est supérieur (35\% ; Bernadet et al communication personnelle). Ces taux sont nettement plus faibles que ceux-observés chez les lapins $(>60 \%)$ mais légèrement plus élevés que ceux observés en production porcine $(20 \%)$ et similaires aux autres volailles (28\% ; Pothin et al 2017). Les principaux coproduits valorisés par les oies sont le son de blé, les drêches de blé ou de maïs et le gluten de maïs, ainsi que les tourteaux de tournesol, de colza ou de soja. En revanche, les oies étant gavées avec une pâtée composée de $59 \%$ de maïs, de $39 \%$ d'eau et de $2 \%$ de prémix, aucun coproduit n'est valorisé pendant cette phase. Sachant qu'une oie prête à gaver ingère en moyenne $22 \mathrm{~kg}$ d'aliment durant sa vie (Ároyo et al 2012c), on peut estimer que, dans le Périgord, l'élevage des oies pour la production de foie gras (130000 animaux) permet de valoriser 77 tonnes de coproduits par an.

\section{2 / Maintien et préservation de la biodiversité}

La préservation de la biodiversité fait aujourd'hui partie des grands défis environnementaux à relever (Convention on Biological Diversity, 2005). Le secteur agricole doit être impliqué dans les efforts à fournir. D'une part, la biodiversité est la source de nombreux services rendus à l'agriculture (Altieri 1999) : auxiliaires de culture, pollinisation, etc. D'autre part, la spécialisation des systèmes de culture et l'intensification des pratiques des dernières décennies amènent à une simplification des paysages agricoles, fortement impliquée dans le déclin de la biodiversité (Burel et al 2008) notamment entomologique.

Une première évaluation de la diversité entomologique présente sur les parcours agroforestiers pour palmipèdes a été réalisée par Bijja et al (2015). Soixante-six collectes ont été réalisées à l'aide de pièges à interception permettant de récolter 5371 individus répartis dans 169 morphotypes. On retrouve majoritairement des homoptères (cicadelles, pucerons...) qui représentent $23 \%$ des individus collectés. Leur abondance est variable au cours de l'année, en raison de l'émergence en masse de pucerons à certaines périodes de l'année. Les collemboles $(14 \%)$, qui sont largement impliqués dans la dégradation de la matière organique, les diptères (mouches, moustiques, tipules ; 14\%) et les hyménoptères (abeilles, guêpes ; 14\%), dont la plupart sont des pollinisateurs, et les coléoptères (carabes ; 11\%), dont beaucoup sont nécrophages ou coprophages, sont représentés dans des proportions similaires. Les autres ordres d'insectes sont plus anecdotiques en termes d'abondance. Les araignées ont représenté $7 \%$ des individus capturés.

L'influence sur la diversité entomologique de la présence d'animaux sur les parcours arborés est présentée dans le tableau 4 pour deux sites de mesures. Il ressort que la diversité entomologique, estimée par l'abondance et la richesse, est très variable entre les sites. Sur le site 1, le parcours accueillant des palmipèdes présente une abondance 10 fois plus élevée (1 660 vs 177 individus) et une richesse 2,5 fois plus élevée (122 vs 52 morphotypes) que le parcours sans animaux. Il est intéressant de noter que les araignées et les lépidoptères (papillons) sont plus nombreux dans les parcours sans animaux que dans les parcours fréquentés. En revanche, sur le deuxième site d'observation, les résultats semblent opposés puisque le parcours sans animaux présente une richesse et une abondance environ deux fois plus élevées que le parcours avec animaux.

Par ailleurs, Bijja et al (2017) ont montré que sur les parcours de palmipèdes, l'activité des lombriciens, espèces-clé de la fertilité du sol, est proportionnelle à l'importance de la fréquentation des animaux dans la zone. Ainsi, $48 \%$ des lombrics anéciques (population majoritaire représentant près de $90 \%$ des individus présents) se trouvent à proximité du bâtiment d'élevage, $34 \%$ dans la zone intermédiaire entre le bâtiment et le fond du parcours et $17 \%$ en fond de parcours. D'autres population de lombrics ont 
Tableau 4. Effets de la présence d'animaux sur la biodiversité entomologique ${ }^{(1)}$ des parcours arborés (d'après Bijja et al 2015).

\begin{tabular}{|l|c|c|c|c|}
\hline Site & \multicolumn{2}{|c|}{ Site 1 } & \multicolumn{2}{c|}{ Site 2 } \\
\hline Zone & $\begin{array}{c}\text { Zone sans } \\
\text { animaux }\end{array}$ & $\begin{array}{c}\text { Zone avec } \\
\text { animaux }\end{array}$ & $\begin{array}{c}\text { Zone sans } \\
\text { animaux }\end{array}$ & $\begin{array}{c}\text { Zone avec } \\
\text { animaux }\end{array}$ \\
\hline Abondance (nombre d'individus) & 177 & 1660 & 2383 & 1151 \\
\hline Richesse (nombre de morpho types) & 52 & 122 & 100 & 47 \\
\hline Richesse recalculée $^{(2)}$ & 52,0 & 51,7 & 27,8 & 24,8 \\
\hline
\end{tabular}

${ }^{(1)}$ Méthode RBA (Oliver et Beattie 1993).

${ }^{(2)}$ Estimée par raréfaction (logiciel EcoSim) d'après Gotelli et Enstminger (2004) pour une abondance fixée à 177.

Tableau 5. Effet de la présence des oies sur la fertilité des sols arborés (d'après Bijja et al 2010).

\begin{tabular}{|c|c|c|c|}
\hline & Témoin sans oie ${ }^{(1)}$ & $\begin{array}{c}\text { Zone peu fréquentée } \\
\text { par les oies }\end{array}$ & $\begin{array}{c}\text { Zone très fréquentée } \\
\text { par les oies }{ }^{(1)}\end{array}$ \\
\hline \multicolumn{4}{|c|}{ Matières organiques (g/kg ${ }^{-1}$ de terre) } \\
\hline Année $\mathrm{n}+1$ & 58,7 & - & 58,6 \\
\hline Année $n+3$ & 57,8 & - & 65,7 \\
\hline \multicolumn{4}{|c|}{ Azote total (g/kg ${ }^{-1}$ de terre) } \\
\hline Année $\mathrm{n}+1$ & 2,5 & 2,6 & 2,7 \\
\hline Année $n+3$ & 2,5 & 2,8 & 2,9 \\
\hline \multicolumn{4}{|l|}{ Rapport C/N } \\
\hline Année $n+1$ & 13,8 & 12,3 & 12,8 \\
\hline Année $\mathrm{n}+3$ & 13,3 & 13,8 & 12,9 \\
\hline \multicolumn{4}{|c|}{ Phosphore, $\mathrm{P}_{2} \mathrm{O}_{5}\left(\mathrm{~g} / \mathrm{kg}^{-1}\right.$ de terre) } \\
\hline Année $n+1$ & 0,58 & 0,63 & 0,80 \\
\hline Année $n+3$ & 0,49 & 0,77 & 0,94 \\
\hline \multicolumn{4}{|c|}{ Potassium, $\mathrm{K}_{2} \mathrm{O}$ (g/ $\mathrm{kg}^{-1}$ de terre) } \\
\hline Année $\mathrm{n}+1$ & 0,40 & 0,45 & 0,62 \\
\hline Année $n+3$ & 0,47 & 0,50 & 0,80 \\
\hline \multicolumn{4}{|c|}{ Magnésium, $\mathrm{MgO}$ (g/kg ${ }^{-1}$ de terre) } \\
\hline Année $n+1$ & 0,22 & 0,21 & 0,23 \\
\hline Année $n+3$ & 0,19 & 0,20 & 0,23 \\
\hline
\end{tabular}

${ }^{(1)}$ Les données rapportées correspondent à la quatrième année de présence ou non des animaux.

également été identifiées : les endogées et les épigés ( 8 et $3 \%$ de la population totale, respectivement). Ainsi, la présence de palmipèdes est favorable à l'activité biologique dans le sol, en particulier au développement des populations lombriciennes.

\section{3 / Maintien de la fertilité des sols}

Pendant leur temps de présence sur les parcours, les oies émettent des déjections riches en éléments fertilisants qui peuvent améliorer la fertilité des sols. L'influence de la présence des animaux sur la fertilité des sols est reportée dans le tableau 5. On note ainsi, qu'en l'absence d'animaux, le taux de matière organique d'un sol arboré évolue peu $\left(58\right.$ g. $\left.\mathrm{kg}^{-1}\right)$. En revanche, la présence d'oies sur le parcours entraine une augmentation notable du taux de matière organique $\left(58,6\right.$ à $\left.65,7 \mathrm{~g} \cdot \mathrm{kg}^{-1}\right)$. Cela représente des quantités de matières organiques de l'ordre de 187 t.ha $^{-1}$ tandis que le seuil moyen pour un sol argilocalcaire est de 90 t.ha-1 (Citeau et al 2008).
Il a été montré qu'un noyer exporte annuellement en moyenne $0,55 \mathrm{~kg}$ d'azote, dont $0,22 \mathrm{~kg}$ qui restent dans la noyeraie (feuilles, brou, branchages) et $0,33 \mathrm{~kg}$ qui sortent de la noyeraie (fruits : coques et cerneaux). Cela représente approximativement 97,5 kg d'azote par hectare pour une noyeraie traditionnelle à 177 arbres par hectare (Gropierre et al 1982). Pour satisfaire ces exportations, une noyeraie traditionnelle nécessite un apport azoté annuel moyen de 74 unités d'azote (Prunet et al 2003). Les résultats du tableau 5 montrent que la présence des oies sur un parcours arboré augmente la teneur en azote du sol, de manière proportionnelle à l'intensité de passage des animaux. Ainsi, sur la zone de forte fréquentation (zone de passage, évaluée par la méthode de scan sampling), la teneur en azote du sol augmente de $16 \%$ en 4 ans $\left(2,5\right.$ g. $\mathrm{kg}^{-1}$ en 2006 vs 2,9 g. kg-1 en 2009) tandis que la zone témoin, sans oies, présente des valeurs d'azote total dans le sol stable et inférieures aux autres zones pour les 4 années. La zone arborée avec oie, mais hors zone de passage présente des valeurs légèrement plus fortes que la zone sans animaux
(2,7 vs 2,5 g. $\left.\mathrm{kg}^{-1}\right)$ mais qui restent stables au cours du temps. Cela suggère que sur cette zone les exportations sont similaires aux apports et que le sol est peu sensible au lessivage. La présence d'animaux sur un parcours arborés est donc favorable à la fertilité du sol et peut permettre d'éviter l'utilisation de fertilisants azotés.

Toutefois, l'azote total ne fournit pas de renseignement sur l'azote minéral disponible pour le végétal. Celui-ci s'évalue essentiellement au travers du rapport $\mathrm{C} / \mathrm{N}$ (carbone organique/azote total) dont les valeurs doivent idéalement se situer entre 9 et 13 (Prunet et al 2003). Les rapports $\mathrm{C} / \mathrm{N}$ de la zone arborée fréquentée par les oies ont évolué de 11,8 à 13,8 en 4 ans ce qui traduit une bonne activité biologique et une bonne minéralisation des éléments fertilisants. Ceci explique probablement les bonnes performances de la noyeraie en termes de croissance des arbres et de production de fruits (voir $\S 2.1 . a$ ).

Contrairement à ce qui est observé pour l'azote, le noyer possède une faible 
capacité d'exportation du phosphore : $0,058 \mathrm{~kg}$ par noyer soit $10,43 \mathrm{~kg} \cdot \mathrm{ha}^{-1}$ (Gropierre et al 1982). Nos résultats montrent que les quantités de phosphore présentes dans le sol, sous forme d'acide phosphorique, sont élevées et se situent au-dessus des seuils d'exigence culturaux normalement observés (Antoni 2009). Les concentrations en phosphore sur la période sont croissantes pour le parcours fréquenté par les animaux, en particulier sur la zone de passage des oies, tandis qu'elles sont stables pour la zone témoin sans animaux qui montre même une tendance à la baisse $(-15 \%)$. Cela suggère, que les apports par les déjections animales sont supérieurs à l'exportation par les arbres mais aussi que le sol est peu sensible au lessivage.

Les seuils habituels de potassium dans le sol se situent entre 0,30 g. $\mathrm{kg}^{-1}$ et 0,45 g. $\mathrm{kg}^{-1}$ de terre. Dans notre étude, la teneur du sol en potassium est plus élevée sur la zone avec présence d'animaux que sur la zone témoin sans oies, plus particulièrement sur la zone de passage des oies $(+70 \%: 0,8$ g.kg-1 vs 0,47 g.kg-1) qui voit ses concentrations potassiques en constante augmentation ( $+21 \%$ sur quatre ans). Toutefois, ces teneurs en potassium du sol suggèrent aussi, qu'en l'absence d'animaux, la présence d'un couvert végétal sur un sol très faiblement lessivable permet une stabilité de la teneur en potassium et ne nécessite aucun apport minéral.

Concernant la teneur en magnésium (tableau 5), nos résultats, compris entre 0,113 g.kg-1 et 0,208 g. $\mathrm{kg}^{-1}$ de terre, montrent que le sol ne présente pas de carence. Les concentrations en magnésium varient peu au cours du temps et ne sont pas influencées par la présence des animaux. Les apports par les animaux sont donc faibles et les exportations en magnésium sont essentiellement concentrées dans les feuilles, dont au moins une partie retourne au sol (Grospierre et al 1982).

\section{4 / Les services patrimoniaux et de qualité de vie}

\section{1 / Esthétique du paysage}

Le paysage, en tant qu'agencement de formes dans un espace limité (un " pays »), a une dimension esthétique forte. Il peut être contemplé à l'horizontale comme à la verticale par un observateur et implique de ce fait un point de vue. Il est généralement considéré comme la résultante de l'action conjointe des sociétés humaines, du monde vivant (animal, végétal, fongique) et du milieu abiotique. La réalisation d'une analyse scientifique du paysage est critiquée par ceux qui
Figure 1. Photo aérienne du site expérimental sur lequel ont été réalisées les mesures avant la plantation des arbres (photo 1) et 11 ans après la plantation (photo 2).
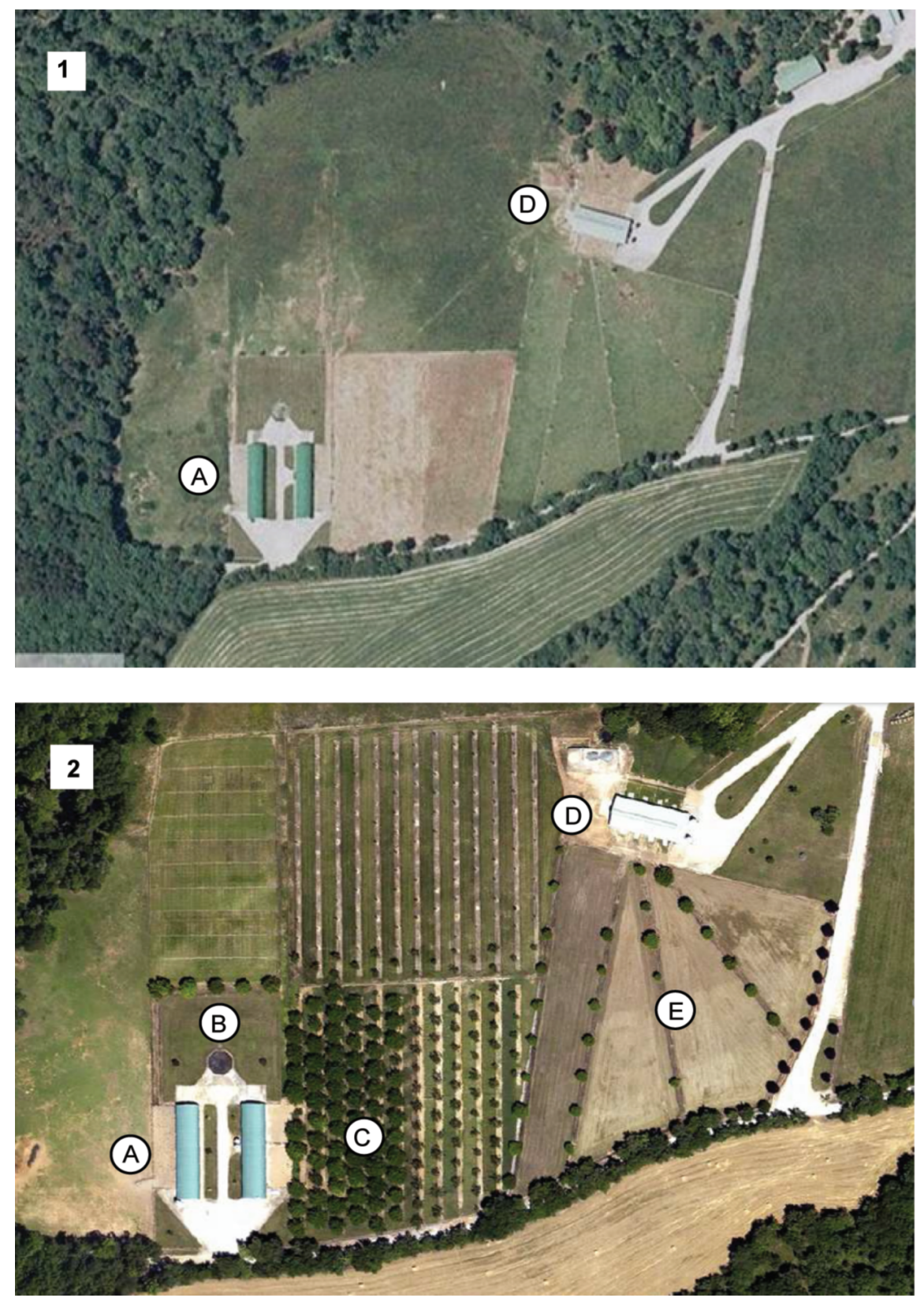

Bâtiments d'élevage (A) avec parcours herbager (B) ou parcours agroforestier planté de noyers (C) ; Bâtiment d'élevage (D) avec parcours herbagers bordés d'arbres (E).

mettent la subjectivité au cœur de l'approche du paysage. Pour autant, il a été montré, par la mesure des réactions psychophysiologiques de personnes placées devant un écran avec différents paysages, que certains paysages ont un effet calmant et agréable (dont les paysages de forêts, d'eau ou de montagnes ; Chang 2004). Ainsi, il a été montré que la naturalité d'un paysage, sa richesse et son harmonie, ainsi que la présence de l'arbre dans le paysage jouent aussi un rôle important dans le sentiment de paix ou de bien-être qu'il procure (Miyake et al 2004). Par ailleurs, il a été montré que l'élevage traditionnel contribue à la création de paysages reconnus comme culturels (Dumont et al 2016). De ce point de vue, la présence de noyers sur les parcours d'élevage des oies pour la production de foie gras contribue assurément à l'esthétique du paysage. L'évolution paysagère de la FEOC suite à la plantation des arbres sur les parcours des animaux est illustrée dans la figure 1 . On peut constater que la présence des arbres apporte de la diversité et de la rugosité, par opposition à la monotonie, sans toutefois fermer le paysage.

\section{2 / Patrimoine culturel et gastro- nomique}

L'oie est très présente dans la culture populaire (expressions, proverbes, dictons, jeu de l'oie), dans l'art (films, séries 
télévisées) et dans la mythologie (les fables d'Ésope légende, la légende des oies du capitole). Mais c'est dans la gastronomie qu'il occupe la place la plus importante aujourd'hui. En effet, le foie gras est inscrit au patrimoine culturel et gastronomique français (article L. 65427 du code rural et article 74 de la loi d'orientation agricole du 5 Janvier 2006). La loi lui donne ainsi une définition légale : « on entend par foie gras, le foie d'un canard ou d'une oie spécialement engraissé par gavage » et autorise la pratique du gavage en France. Ce texte permet de reconnaitre que cette spécificité culinaire française est ancrée dans les traditions régionales et contribue à en faire un ambassadeur de la culture française.

Afin de défendre la qualité et l'origine des produits issus de palmipèdes à foie gras du Périgord et d'offrir plus de transparence aux consommateurs, des éleveurs, découpeurs et conserveurs de Dordogne se sont regroupés en créant en 1993 l'Association Foie Gras du Périgord (http:// www.foiegras-perigord.com/). Celle-ci a créé une Indication Géographique Protégée (IGP) « Canard à Foie Gras du Périgord » et la marque Oie du Périgord déposée à l'INPI et validée en 2012, tandis que l'IGP « Oie du Périgord » est déposée mais n'est pas encore validée administrativement. Ce cahier des charges garantie que les produits sont issus d'animaux élevés, gavés, abattus, découpés, cuisinés et conditionnés en Périgord et fait du «Foie gras du Périgord » un produit à haute valeur ajoutée.

Par ailleurs, le Périgord bénéficie de l'AOC " Noix du Périgord » depuis 2002 pour la noix fraîche, la noix sèche et le cerneau de noix (1 $700 \mathrm{t}$ de noix en $2014 ;$ http://www.noixduperigord.com). Ces produits sont issus de 4 variétés locales : la Corne (11\%), la Marbot (12\%), la Grandjean (4\%) et la Franquette (70\%). Dans le cahier des charges de l'AOC il est spécifié que la densité de plantation est de 125 arbres/ha maximum à partir de la $15^{\text {ème }}$ année et la distance minimale entre les noyers doit être au moins égale à 7 mètres. Nos résultats (voir $\S 2.2$.), obtenus sur une noyeraie contenant 156 arbres /ha (distance entre les noyers de $8 \mathrm{~m}$, voir $\S 1.2$.), montrent que cette disposition est tout à fait compatible avec l'utilisation de la zone arborée pour le parcours des palmipèdes.

\section{3 / Liens socio-éducatifs}

Les apports socio-éducatifs des animaux ont beaucoup été décrits pour les animaux de compagnie. Ceux-ci ont été démontrés comme étant des vecteurs de communication et d'intégration sociale. La contribution de l'élevage est complémentaire. L'animal permet ici à l'Homme de conserver, voire de renouer un lien avec la nature. L'élevage rappelle ainsi à l'Homme la nécessité de respecter les rythmes naturels du cycle de vie de l'animal, ainsi que ses besoins fondamentaux d'exercice, de communication et d'alimentation, etc. Lorsque les animaux sont élevés en confinement total, ce qui peut être le cas des animaux monogastriques, ce lien à l'animal peut se distendre car les animaux ne sont plus visibles aux hommes autres que les éleveurs. De ce point de vue, les parcours pour les volailles présentent, outre un intérêt pour les animaux, mais également pour l'Homme, car ils permettent de remettre du contact entre l'Homme et les animaux d'élevage. Dans cet objectif, nous nous sommes attachés à quantifier, l'intérêt d'un aménagement forestier du parcours sur le comportement des oies.

Nous avons ainsi pu montrer que les jours ensoleillés, les oies se dirigent majoritairement sous l'ombre des noyers, quelle que soit l'heure de la journée (figure 2 ; Dubois et al 2008). Ainsi, le taux de présence des oies à l'ombre varie de $69 \%$ à $9 \mathrm{~h}$ du matin, à $96 \%$ à $14 \mathrm{~h}$ et $87 \%$ à $17 \mathrm{~h}$. Durant les jours chauds (plus de $25^{\circ} \mathrm{C}$ ), les oies n'utilisent que très peu le bâtiment (de 1 à $3 \%$ des animaux suivant les heures de la journée), pourtant légèrement plus frais que l'extérieur, et préfèrent rester couchées à l'ombre des arbres que dans le bâtiment qui est en libre accès. D'ailleurs, à 14 h, il n'y a aucun animal au soleil dans la zone non ombragée.

Lorsque les jours sont plus frais $\left(<20^{\circ} \mathrm{C}\right)$ et/ou nuageux, les oies vont plus facilement dans le bâtiment, surtout en fin de journée (figure 3). Ainsi, elles se répartissent plus sur la zone enherbée non ombragée, surtout en début de journée (63\% des oies à $9 \mathrm{~h}$ ). Toutefois, en milieu de journée (14 h), elles se répartissent de façon quasiment équitable entre la zone ombragée $(59 \%)$ et non ombragée $(37 \%)$. Enfin, en fin de journée $(17 \mathrm{~h})$, les oies se rapprochent du bâtiment et sont $76 \%$ sur l'espace caillouteux contre le bâtiment et $22 \%$ dans le bâtiment.

\section{5 / Les services de vitalité territoriale}

\section{1 / Vitalité des filières}

La filière foie gras génère 100000 emplois directs et indirects en France et génère environ deux milliards d'euros de chiffre d'affaire (sortie entreprise ; CIFOG 2015). C'est donc une filière très importante pour la vitalité territoriale au niveau national. De ce point de vue, la filière foie gras d'oie du Périgord est extrêmement modeste. Le foie d'oie représentant moins de $5 \%$ de la production de foie gras nationale. En revanche, le Périgord possède la particularité de

Figure 2. Répartition des oies sur le parcours arboré par journée pluvieuse (A) ou ensoleillée (B).
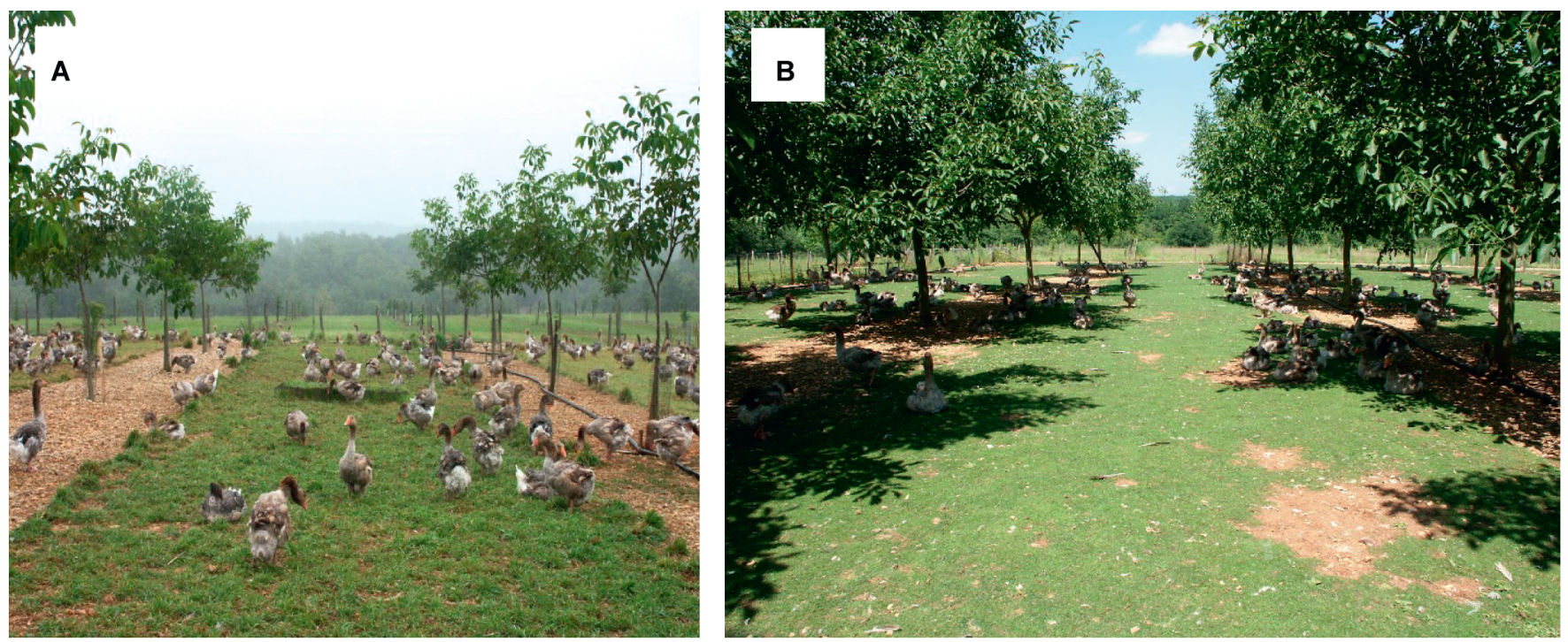
Figure 3. Évolution de la répartition des oies sur l'ensemble de l'espace disponible dans la zone d'élevage au cours de la journée et en fonction de la météorologie (journée pluvieuse $(A)$ ou journée ensoleillée $(B)$; $\mathrm{n}=4$ observations par situation ; adapté de Dubois et al 2008).

A

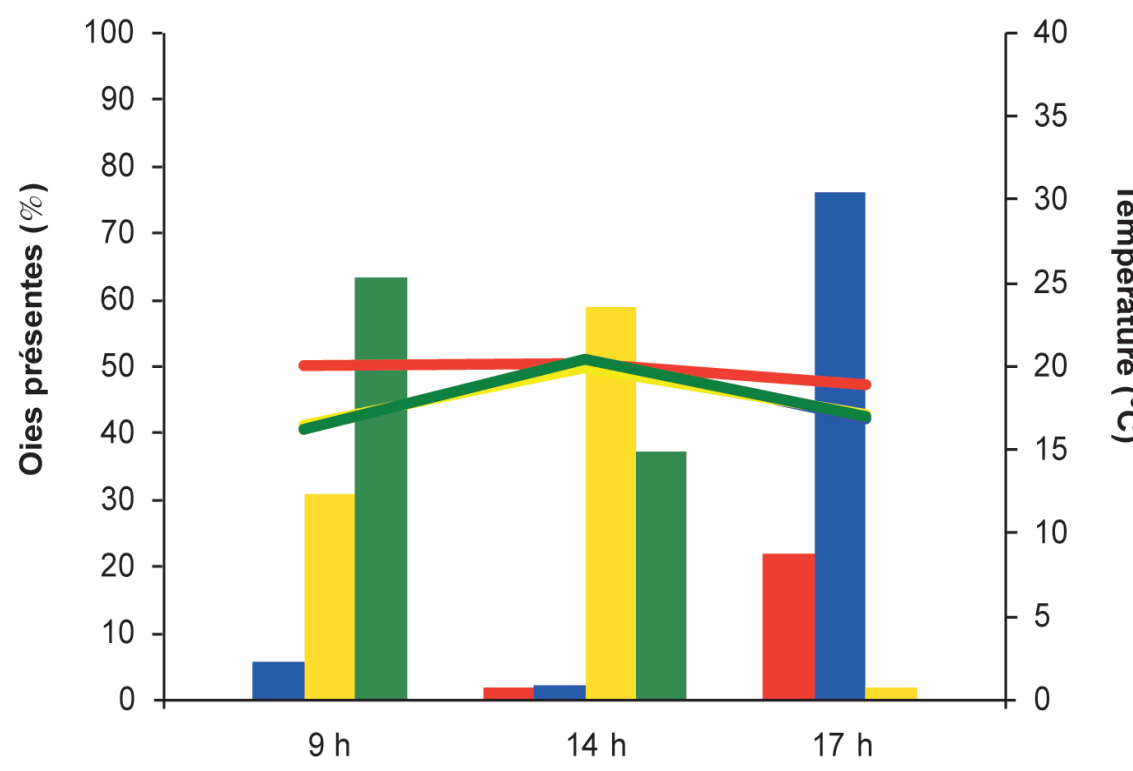

B

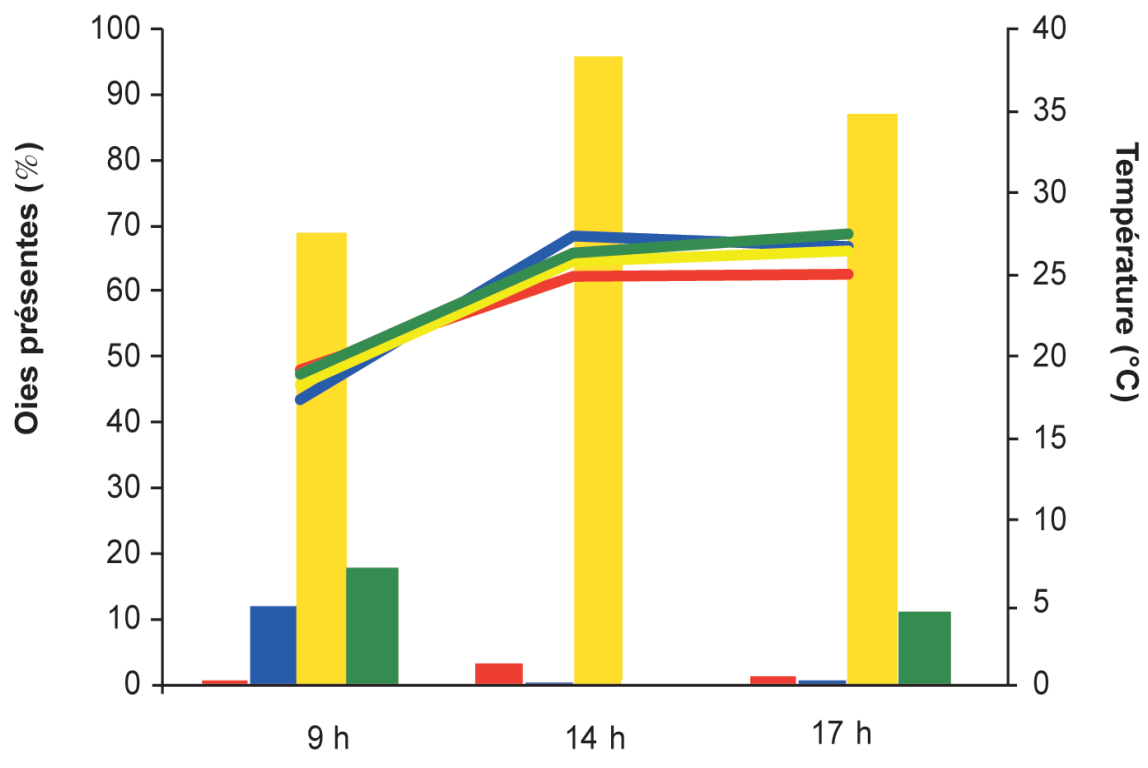

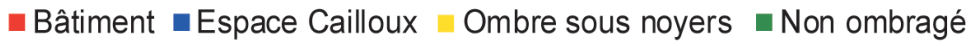

regrouper sur son territoire la totalité des maillons de la filière « oie » : reproduction, accouvage, élevage, gavage, abattage et transformation. Le nombre d'emplois directs généré pour la filière « oies grasses en Périgord » est difficile à estimer car de nombreux producteurs d'oies élèvent également des canards. Toutefois, les travaux de Farrant et al (2017) montrent pour la production de foie gras de canards IGP du sud-ouest, la production d'une tonne de foie gras créée 5,77 emplois au niveau de toute la filière (distribution et commercialisation comprises). En conséquence, si l'on garde ce même ratio, on peut estimer que les marge nette de $12,7 € /$ oie, ce qui est plus important qu'en filière longue (34 $€ /$ oie et 3,5€/oie, respectivement) ou en filière courte de canard gars $(28$ à $69 € /$ canard et 5 à $13,7 € /$ canard, respectivement suivant le type de d'organisation : familiale, en groupe...). De plus, elle génère un nombre important d'emplois dans les exploitations agricoles puisque les ateliers en filière courte emploient en moyenne 1,9 UTH dont 1,3 UTH salariés (Litt 2015).

\section{3 / Vitalité rurale}

L'agrotourisme, appelé aussi tourisme agricole ou tourisme à la ferme, est une forme de tourisme dont l'objet est la découverte des savoirs-faire agricoles d'un territoire, et par extension des paysages, des pratiques sociales et des spécialités culinaires découlant de l'agriculture. Cette activité, qui permet de générer un développement économique plus ou moins marqué pour les territoires et les agriculteurs concernés, est en plein développement. Ainsi, on recense en France près de 14000 exploitations agricoles qui exercent des activités liées au tourisme. Cette activité prend des formes multiples répondant aux attentes des touristes : un accueil limité à l'habitat (les gîtes ruraux), un accueil intégrant les repas à la ferme même (chambres d'hôtes), et l'intégration à un certain nombre d'activités, comme la production, ou la vente de produits sur place.

L'agrotourisme est particulièrement développé dans le Périgord. Ainsi, la Dordogne est le 10 ème département français le plus fréquenté par les touristes où l'agrotourisme y est très développé. La ruralité de ce département, ainsi que les produits à forte valeur ajoutée, tel que le foie gras, ou ceux ayant une forte identité territoriale, telle que les noix du Périgord, sont clairement des éléments qui expliquent cette situation. Le site internet http://www.guide-du-perigord.com/fr/ recense i) 74 adresses permettant de déguster du foie gras, dont 10 mentionnent spécifiquement le foie d'oie, et 12 adresses pour la dégustation de la noix et de ses dérivés ; ii) 183 exploitations agricoles réalisant l'accueil à la ferme ; iii) 28 fermes auberges et 5 tables d'hôtes et iv) 47 marchés de producteurs et 4 magasins de producteurs qui font tous la part belle au foie gras d'oie.

\section{2 / Vitalité des exploitations agricoles}

Au niveau des exploitations agricole, l'élevage des oies grasses est une production agricole qui permet un revenu très décent (36000 $€$ de revenu disponible/UTHf / an pour les producteurs de foie gras d'oie ; Litt 2015). En effet, en filière courte (majorité des cas en Périgord), une oie grasse est valorisée $94,5 € /$ oie et permet de dégager une

\section{6 / Les dys-services}

\section{1 / Acceptabilité sociale}

La production de foie gras est aujourd'hui obtenue par alimentation forcée des animaux avec introduction de l'aliment directement dans le jabot (acte d'embu- 
cage). Cette pratique est sujette à controverse. D'un côté, les associations de protection animale considèrent qu'elle est contraire au bien-être animal même si la stéatose hépatique d'origine nutritionnelle est réversible (Babilé et al 1998) et que l'absence d'élévation du taux de corticostérone après l'acte de gavage (Guémené et al 2007) suggère que l'acte d'embucage ne génère pas de stress aigu chez les animaux. Pour autant, en 2014, 95\% des français ont déclaré être consommateurs de foie gras et le foie gras reste le produit incontournable des fêtes (devant le saumon fumé ou la bûche de noël) pour $79 \%$ des français (enquête CIFOG/CSA réalisée en 2014 ; http:// lefoiegras.fr). C'est dans ce contexte, que la commission européenne a interdit depuis 1999 la production de foie gras dans tous les pays européens n'étant pas déjà producteurs. Seuls les pays producteurs à cette date ont été autorisés à poursuivre la pratique du gavage à condition toutefois qu'ils démontrent qu'ils sont actifs dans la recherche d'alternatives au gavage (Recommandation européenne du 22 juin 1999, article 24). La France, premier pays producteur mondial de foie gras, a engagé des recherches dans ce sens depuis 2009. Une première preuve de concept a été démontrée chez l'oie (Guy et al 2013). Toutefois, les connaissances actuelles ne permettent pas de proposer un système de production économiquement viable ni socialement acceptable (Knudsen et al 2017).

\section{2 / Les impacts environnementaux}

La plupart des activités humaines génèrent des impacts environnementaux potentiels qui peuvent être quantifiés par la méthode de l'analyse des cycles de vie. Arroyo et al (2013c) ont montré que la production de $1 \mathrm{~kg}$ de foie de foie gras d'oie génère un potentiel d'acidification $(0,52 \mathrm{~kg} \mathrm{SO}$-eq. $/ 1 \mathrm{~kg})$ et une occupation des surfaces agricoles $\left(48,6 \mathrm{~m}^{2} \mathrm{an} /\right.$ $1 \mathrm{~kg}$ ) équivalent à celle de la viande de bœuf mais supérieure à celle des produits issus d'animaux monogastriques (œuf, viande d'oie, de volaille ou de porc) ou de la production de lait (de Vries and de Boer 2010 ; Leinonen et al 2012a, $2012 b)$. Elle est la production animale qui génère le plus de réchauffement climatique potentiel par $\mathrm{kg}$ de produit $(37,5 \mathrm{~kg} \mathrm{CO}$-eq./1 kg) Cela est expliqué par le fait que les oies sont élevées presque jusqu'à la puberté et que le produit est gras, la quantité de ressources nécessaires pour obtenir le produit est donc très élevé. D'ailleurs, les impacts environnementaux potentiels de la production de foie gras de canards sont inférieurs à ceux de la production de foie gras d'oie (Deneufbourg et al 2017) en raison d'une durée d'élevage plus courte et d'une meilleure valorisation écono- mique de la viande de canard par le biais des magrets (principe de l'allocation des ressources entre les divers coproduits).

Parallèlement, Cambria et Pierangeli (2012) ont montré que la production de $1 \mathrm{~m}^{3}$ de bois de noyers génère un potentiel de réchauffement climatique de $110 \mathrm{~kg}$ $\mathrm{CO}_{2}$-eq., une eutrophisation potentielle de $580 \mathrm{~kg} \mathrm{PO}_{4}$-eq., une acidification potentielle de $770 \mathrm{~kg} \mathrm{SO}_{2}$-eq. et une écotoxicité terrestre de $46,8 \mathrm{~kg}$ 1.4-DCBeq. C'est la phase de plantation qui génère près de $90 \%$ des impacts, les opérations sylvicoles et le transport expliquant les effets restant. En effet, la croissance des arbres en elle-même ne génère aucun impact et est même bénéfique d'un point de vue environnemental. En effet, les forêts entraînent des absorptions de $\mathrm{CO}_{2}$ (biomasse vivante, biomasse morte et le sol), et le protocole de Kyoto identifie les forêts comme un des leviers d'action pour réduire l'émission des gaz à effet de serre d'origine humaine.

Il n'existe pas, à notre connaissance, d'étude concernant les conséquences d'une association oies/noyers sur les impacts environnementaux potentiels. Mais Smith et al (2013) ont montré que l'établissement d'arbres sur les terres agricoles intégrés avec des cultures et / ou une production animale peut aider à atténuer bon nombre des impacts négatifs de l'agriculture. Nous pensons que cela sera le cas également pour l'association oies/noyers puisque la fertilisation des arbres par les déjections des oies augmente la production obtenue (bois, fruits) sans augmentation des ressources nécessaires.

\section{3 / Sécurité sanitaire}

En termes sanitaires, c'est avec l'influenza aviaire, plus connue sous le nom de grippe aviaire, que l'élevage d'oie à le plus de problèmes et peut poser le plus de problèmes aux autres filières d'élevage. Cette maladie concerne les oiseaux domestiques et sauvages et est classée danger sanitaire de première catégorie. Ce virus présente de nombreux variants, de pathogénicité diverses (ex : H5N1, H5N2, H5N8, H5N9...) dont la plupart ne sont pas transmissibles à l'Homme ni à d'autres mammifères. Une sévère épidémie de grippe aviaire sévit en France depuis la fin de l'année 2015 touchant majoritairement des élevages de palmipèdes gras. Les palmipèdes à foie gras, dont l'oie, sont considérés comme d'importants réservoirs pour certains variants de cet agent pathogène car ces animaux, contrairement aux gallinacés, sont naturellement peu sensibles et peuvent héberger le virus en présentant pas ou peu de signes cliniques (Le Bouquin et al 2016).
De manière plus spécifique à l'association oies/noyers, il semblait important de vérifier que l'association animal/ arbre fruitier ne génère pas de contaminations bactériologiques indésirables sur les fruits. Pour cela, nous avons réalisé des analyses de portage de Salmonelle et de $E$. coli sur des noix issues des parcours plantés de noyers ayant eu ou non la présence d'oies (Bijja et al 2008). Les noix ont été récoltées 27 jours après l'évacuation des animaux ou, sur un lot de fruits, en présence des animaux (tableau 6). Les analyses montrent que la qualité sanitaire de tous les lots de cerneaux lavés et séchés répond aux critères de sécurité, conformément au règlement $\mathrm{CE} \mathrm{N}^{\circ} 2073$ / 2005 concernant les fruits prêts à être consommés. Nos résultats ont montré qu'il peut parfois y avoir un dépassement de ces recommandations pour E. coli sur les coques avant traitement. Mais cette situation a été observée à la fois pour les coques de noix issus de zone avec présence d'oies (lots 2, 3, 5 et 6 du tableau 6) ou sans oies (lot 4 avec 140 ufc E. Coli/g), et ce, malgré les précautions sanitaires prises lors de la récolte. Ces résultats montrent qu'un risque sanitaire existe si les noix sont récoltées en présence d'animaux (lot 7), même si le lot de cerneaux concerné s'est révélé indemne après séchage (tableau 6). En conséquence, le délai de 27 jours que nous avons appliqué entre le passage des animaux et la récolte semble être un minimum. La station de recherche sur la noix de Creysse (46) conseille un délai de 2 mois (Prunet et al 2003).

\section{7 / Qualification du bouquet de services et leviers d'actions}

Nous avons cherché à qualifier les bouquets de services rendus par le système d'élevage agroforestier d'oie avec des noyers sur parcours (contribution faible, moyenne ou forte ; figure 4) afin décrire la pluralité des rôles et des impacts issus de l'activité d'élevage.

Le foie gras et les noix ne sont pas des matières premières qui constituent une part importante de notre alimentation. Ainsi, la consommation moyenne de foie gras est de $276 \mathrm{~g}$ par personne/an (mais $657 \mathrm{~g}$ de foie gras acheté par ménage acheteur ; CIFOG, 2015) dont 95\% est du foie gras de canard. Cela représente donc une très faible contribution aux apports alimentaires en produits carnés au regard des $87 \mathrm{~kg}$ équivalent carcasse consommés par personne et par an en France. De même, la consommation française moyenne de noix est de $500 \mathrm{~g}$ par personne et par an ce qui est également très faible au regard des $190 \mathrm{~kg}$ de fruits et légumes consommés par personne et par an (Blot 2012). Notre modèle d'étude 
Tableau 6. Influence de la présence des oies sous les noyers sur la qualité sanitaire des noix (d'après Dubois et al 2008).

\begin{tabular}{|c|c|c|c|c|c|c|c|c|}
\hline \multirow[b]{2}{*}{ Espèce bactérienne } & \multicolumn{4}{|c|}{ Avant lavage } & \multicolumn{4}{|c|}{ Après lavage et séchage } \\
\hline & \multicolumn{2}{|c|}{ Salmonella } & \multicolumn{2}{|c|}{ E. Coli } & \multicolumn{2}{|c|}{ Salmonella } & \multicolumn{2}{|c|}{ E. Coli } \\
\hline Norme & \multicolumn{2}{|c|}{ Absence/25 g } & \multicolumn{2}{|c|}{$\mathrm{UFC}^{(1)} / \mathrm{g}<100$} & \multicolumn{2}{|c|}{ Absence/25 g } & \multicolumn{2}{|c|}{$\mathrm{UFC}^{(1)} / \mathrm{g}<100$} \\
\hline Matrice & Coques & Cerneaux & Coques & Cerneaux & Coques & Cerneaux & Coques & Cerneaux \\
\hline \multicolumn{9}{|l|}{ Echantillon } \\
\hline 1 (zone sans oie) & abs & abs & 20 & $<10$ & abs & abs & $<20$ & $<10$ \\
\hline 2 (zone avec oies) & abs & abs & 280 & $<10$ & abs & abs & $<20$ & $<10$ \\
\hline 3 (zone avec oies) & abs & abs & $<20$ & $<10$ & abs & abs & $<20$ & $<10$ \\
\hline 4 (zone avec oies) & abs & abs & 140 & $<10$ & abs & abs & $<20$ & $<10$ \\
\hline 5 (zone sans oie) & abs & abs & 1340 & $<10$ & abs & abs & $<20$ & $<10$ \\
\hline 6 (zone avec oies) & abs & abs & 700 & $<10$ & abs & abs & $<20$ & $<10$ \\
\hline $\begin{array}{l}7 \text { (prél. réalisé en } \\
\text { présence des oies) }\end{array}$ & abs & abs & 200 & 490 & abs & abs & 180 & $<10$ \\
\hline
\end{tabular}

(1) UFC : Unité Formant Colonie.

Figure 4. Qualification du bouquet de services rendus par le système agroforestier d'élevage d'oie pour la production de foie gras (adapté de Ryschawy et al 2015).

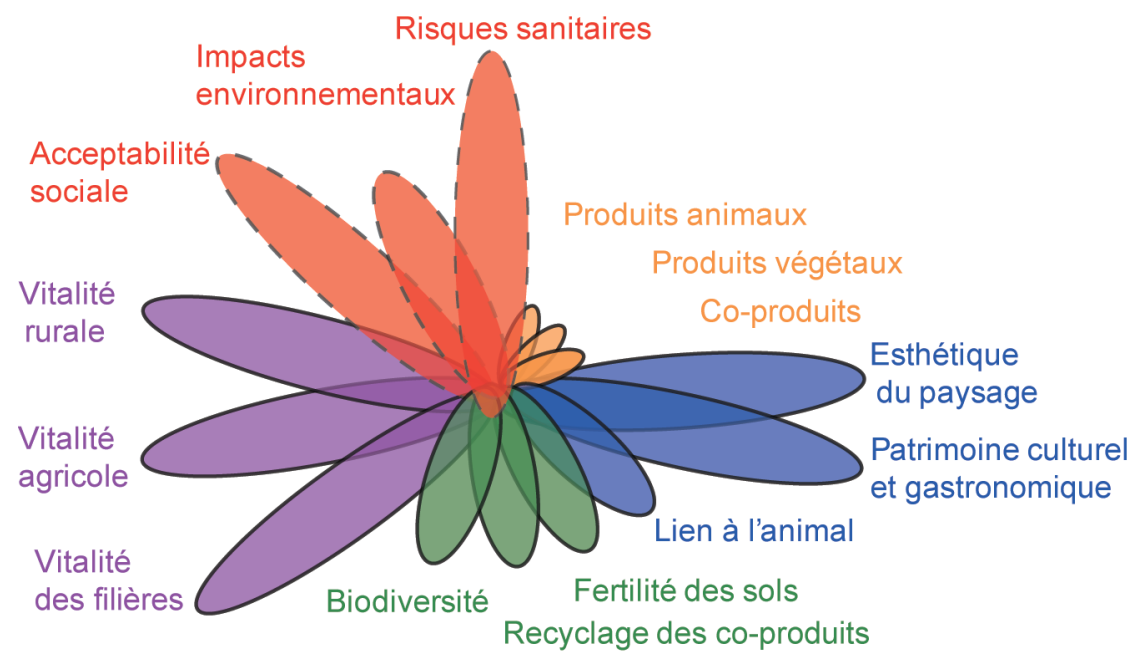

La taille des pétales (petite, moyenne ou grande) symbolise l'importance de la contribution (faible, moyenne ou forte) du système d'élevage à la catégorie de service (trait plein) ou de disservice (trait pointillé).

n'apporte donc pas une contribution majeure aux services d'approvisionnement.

De même, si nous avons montré les bénéfices de l'association oies/noyers sur la qualité environnementale que ce soit la fertilité des sols, la préservation de la biodiversité, la diversité d'utilisation des sols ou la valorisation des coproduits, il est évident que cela reste une contribution quantitativement modeste au regard des surfaces utilisées (SAU moyenne des exploitations produisant du foie gras d'oie : 32 ha; Litt 2015) et des volumes produits (voir $\S 2.2 . \mathrm{a}$ ).

En revanche, cette association originale apporte une contribution significa- tive aux services de vitalité territoriale et de préservation du patrimoine et de la qualité de vie. Comme nous l'avons montré, le Foie gras du Périgord et les Noix du Périgord sont associés à une très forte identité culturelle et/ou gastronomique, reconnue d'un point de vue légale par le biais de produits labellisés et à haute valeur ajoutée. Le Périgord est un territoire présentant une activité d'agrotourisme très développé (gîtes ruraux, chambres d'hôtes, fermes auberges, camping à la ferme, vente directe, marchés festifs...) et le foie gras et ses dérivés y occupent une place non négligeable. Même si la production de foie gras d'oie est en déclin, elle permet, comme tous les produits de niche, une diversification de l'offre alimentaire fes- tive et le foie gras d'oie est le produit le plus haut de gamme de cette filière. Ces services rendus permettent de contrebalancer les disservices en terme de d'impacts environnementaux, de risque sanitaire ou d'acceptabilité sociale.

Les leviers d'action envisageables pour harmoniser le bouquet de services sont divers. Pour réduire les externalités négatives, on peut citer par exemple l'alimentation (choix des matières premières, gestion des transitions, modes de présentation, préparation au gavage) ou la gestion des déjections pour limiter les impacts environnementaux, la conduite d'élevage (dont la recherche d'alternatives au gavage) et/ou le mode de logement des animaux pour améliorer l'acceptabilité sociale de l'élevage, la gestion de l'accès au parcours (notamment pendant les périodes de migration de l'avifaune sauvage) ou l'organisation des filières (modes d'échanges et de transport entre les maillons) pour limiter les risques sanitaires (dont l'Influenza aviaire). Concernant les externalités positives on peut citer par exemple l'aménagement (disposition, introduction d'espèces arbustives en bordures de parcours) et la gestion des parcours pour améliorer les effets positifs sur la qualité des sols et la biodiversité, la gouvernance territoriale pour continuer à valoriser cette spécificité territoriale.

\section{Conclusion}

L'association entre des oies et des noyers est une pratique ancestrale dans le Périgord pratiquée par la majorité des producteurs de foie gras qui se trouvent dans la zone AOC Noix du Périgord. À l'origine son intérêt était principale- 
ment zootechnique, les noyers apportant de l'ombre aux animaux, et économique, les noyers apportant une diversification des sources de revenus (bois, fruits), soutenue par une production accrue grâce aux déjections des oies qui fertilisent les arbres. Notre travail permet de montrer, par une approche basée sur la diversité des services rendus, que les bénéfices de cette association sont beaucoup plus larges et concernent la préservation de l'environnement, de la vitalité rurale, de la qualité des paysages et du patrimoine gastronomique. Dans un contexte où l'élevage est souvent vu principalement sous l'angle des impacts environnementaux qu'il génère, ce type d'approche, basé sur la multifonctionnalité des systèmes de production permet de réfléchir à la complexité des liens qu'entretiennent les systèmes d'élevage au sein de leur territoire. Il invite également à penser aux possibilités de compromis, d'une part entre les services au sein d'un système de production, et d'autre part entre des acteurs aux intérêts différenciés. L'agroforesterie connaissant un regain d'intérêt dans les élevages de volailles en général, et de production de foie gras en particulier, ce travail permettra de contribuer à accompagner la transition écologique des systèmes agricoles.

\section{Références}

Altieri M.A., 1999. The ecological role of biodiversity in agroecosystems. Agricult. Ecosys. Environ., 74, 19-31.

Antoni V., 2009. Le Phosphore dans les sols, nécessité agronomique et préoccupation environnementale. Observation et statistiques, Bul. Techn.,14, http://www.statistiques.developpement-durable.gouv.fr/publications/p/119/ 1097/phosphore-sols-necessite-agronomiquepreoccupation.html

Arroyo J., Auvergne A., Dubois J.P., Lavigne F., Bijja M., Bannelier C., Fortun-Lamothe L. 2012a. Effects of presentation and type of cereals (corn or sorghum) on performance of geese. Poult. Sci., 91, 2063-2071.

Arroyo J., Auvergne A., Dubois J.P., Lavigne F., Bijja M., Fortun-Lamothe L., 2012b. Influence of feeding sorghum on the growth, gizzard development and carcass traits of growing geese. Animal, 6, 1583-1589.

Arroyo J., Fortun-Lamothe L., Dubois J.P., Lavigne F., Auvergne A., 2012c. Conduite et gestion des transitions alimentaires chez les oies destinées à la production de foie gras. INRA Prod. Anim., 25, 419-430.

Arroyo J., Auvergne A., Dubois J.P., Lavigne F., Bijja M., Bannelier C., Manse H., FortunLamothe L., 2013a. Effects of substituting yellow corn for sorghum in geese diets on magret and foie gras quality. Poult. Sci., 92, 24482456.

Arroyo J., Auvergne A., Dubois J.P., Lavigne F., Bijja M., Fortun-Lamothe L., 2013 b. Influence of amount and form of sorghum in the diet on the performance of overfed geese. J. Appl. Poult. Res., 22, 849-854.

Arroyo J., Fortun-Lamothe L., Auvergne A., Dubois J.P., Lavigne F., Bijja M., Aubin J., 2013c. Environmental influence of maize substitution by sorghum and diet presentation on goose foie gras production. J. Clean. Prod., 59, 51-62.

Arroyo J., Brachet, M., Dubois J.P., Lavigne F., Molette, C., Bannelier, C. Fortun-Lamothe L., Auvergne A., 2015. Effect of incorporating sugar beet pulp in the finisher diet on performance of geese. Animal, 9, 553-560.

Aznar O., 2014. Agriculture de service quelques éléments d'analyse économique. 221, 79-86 (doi: 10.3917/pour.221.0079).

Babilé R., Auvergne A., Dubois J.P., Benard P., Manse H., 1998. Réversibilité de la stéatose hépatique chez l'oie. Journ. Rech. Palmipèdes à Foie Gras, 3, 45-48.
Baéza E., Fernandez X., Marie-Etancelin C., 2013. Qualité des carcasses et de la viande des palmipèdes gavés. INRA Prod. Anim., 26, 425434.

Ballard C., 1997. L'envolée sauvage. Fly Away Home. DVD.

Bijja M., Dubois J.P., Guérin J.L., Lavigne F., Auvergne A., Arroyo J., Fernandez X., 2010. Palmipèdes et agroforesterie : performances du verger et évolution de la teneur du sol en éléments fertilisants après 4 années de pratique. Journ. Rech. Palmipèdes à Foie Gras, 9, 245249

Bijja M., Lavigne F., Dubois J.P., Arroyo J., 2015. Impact de l'élevage de palmipèdes à foie gras sur la biodiversité entomologique. 1ère étude. 11 ème Journ. Rech. Avicole et Palmipèdes à Foie Gras.

Bijja M., Lavigne F., Mondoux C., Arroyo J., 2017. Influence du mode de conduite d'élevage des canards mulards sur la fertilité des sols et des populations de lombriciens. 11 ème Journ. Rech. Avicole et Palmipèdes à Foie Gras.

Bliefert C., Perraud R., 2001. Chimie de l'environnement : air, eau, sols, déchets. De Boeck Édition, Université, ISBN : 978-2-8041-5945-0.

Blot C., 2012. Fruits et légumes : évolutions et tendances de consommation. Rapport d'étude de France Agrimer download at http://www.france agrimer.fr/fam/content/download/13422/96341 file/Conf\% C3\% A 9renceFranceAgriMerSIA+Fruits + et $+1 \%$ C $3 \%$ A9gumes $+28+02+201$ 2.pdf

Burel F., Garnier E., Amiaud B., Aulagnier S. Butet A., Chauvel B., Villenave C., 2008. Expertise collective Agriculture et Biodiversité. Chapitre 1. Les effets de l'agriculture sur la biodiversité, download on http://institut.inra.fr/ Missions/Eclairer-les-decisions/Expertises/ Toutes-les-actualites/Agriculture-et-biodiversite

Cambira D., Pierangeli D., 2012. Application of a life cycle assessment to walnut tree (Juglans regia L.) high quality wood production: a case study in southern Italy. J. Cleaner Prod., 23, $37-46$.

CIFOG (Comité Interprofessionnel des palmipèdes à Foie Gras). 2012. Rapport économique 2011. Assemblée Générale du 22/06/12, SaintPalais, France, $75 \mathrm{p}$.

CIFOG (Comité Interprofessionnel des palmipèdes à Foie Gras). 2015. Rapport économique 2014. Assemblée Générale du 24/06/2015. Hossegor, France.
Citeau L., Bispo A., Bardy M., King D., 2008. Gestion durable des sols. Edition Quae, ISBN : 978-2-7592-0189-1.

Chang C.Y., 2004, Psychophysiological responses to different landscape settings and a comparison of cultural differences. ISHS Acta Horticulturae 639: XXVI Int. Horticult. Congr. : Expanding Roles for Horticulture in Improving Human Well-Being and Life Quality.

Convention on Biological Diversity (Secretariat of the) 2005. Handbook of the Convention on Biological Diversity Including its Cartagena Protocol on Biosafety, $3^{\text {rd }}$ Edition, Montréal, Canada), https://www.cbd.int/doc/handbook/ cbd-hb-all-en.pdf

Deneufbourg C., Arroyo J., Wilfart A., Farrant L., Litt J., Fortun-Lamothe L., 2017. Impacts environnementaux de trois systèmes contrastés de production de foie gras de canard. Journ. Rech. Avicole et Palmipèdes à Foie Gras, 12, 1124p.

Dubois J.P., Bijja M., Auvergne A., Lavigne F., Fernandez X., Babilé R., 2008. Agroforesterie: comportement des oies sous un couvert de noyers et effets sur les performances du verger. Journ. Rech. Palmipèdes à Foie Gras. 8, 111-114.

Dumont B., Dupraz P., Aubin J., Benoit M., Bouamra-Mechemache Z., Chatellier V., Delaby L., Delfosse C. Dourmad J.Y., Duru M., Frappier L., Friant-Perrot M., Gaigné C., Girard A., Guichet J.L., Havlik P., Hostiou N., Huguenin-Elie O., Klumpp K., Langlais A., Lemauviel-Lavenant S., Le Perchec S., Lepiller O., Méda, B., Ryschawy J., Sabatier R., Veissier I., Verrier E., Vollet D., Savini I., Hercule J., Donnars C., 2016. Rôles, impacts et services issus des élevages en Europe. Synthèse de l'expertise scientifique collective, INRA, France.

Farrant L., Labau M.P., Padilla M., Deneufbourg C., Fortun-Lamothe L., Penavayre S., Besnier A., 2017. Filière IGP canard à foie gras du sudouest : bilan environnemental, et évaluation des performances sociale, économique et territoriale. 12 ème Journ Rech Avicole et Palmipèdes à Foie Gras.

Gerber P.J., Steinfeld H., Henderson B., Mottet A., Opio C., Dijkman J., Falcucci A., Tempio G., 2013. Tackling climate change through livestock - A global assessment of emissions and mitigation opportunities. Rome: FAO, 115 p. http://www.fao.org/docrep/018/i3437e i3437e.pdf

Grospierre P, Charlot G., Huguet C., Jestin Y., 1982. Nutrition et fertilisation du Noyer. CTIFL, 592,100 p.

Guémené D., Guy G., Mirabito L., Servière J., Faure J.M., 2007. Bien-être et élevage des 
palmipèdes. In : Numéro spécial, Bien-être animal. INRA Prod. Anim., 20, 53-57.

Guy G., Fortun-Lamothe L., Benard G., Fernandez X., 2013. A natural induction of spontaneous liver steatosis in Greylag Landaise geese (Anser anser). J. Anim. Sci., 91, 455-464.

Knudsen C., Guy G., Bonnefont C., FortunLamothe L., Ricaud K., Fernandez X., 2017. L'engraissement spontané du foie chez les palmipèdes : état des lieux et perspectives de recherche. 12 ème Journ. Rech. Avicole et Palmipèdes à Foie Gras.

Le Bouquin S., Huneau-Salaün A., Hamon M., Moisson M.C., Scoizec A., Niqueux E., Schmitz A., Briand F.X., Van De Wiele A., Bronner A., 2016. L'épizootie d'Influenza aviaire en France en 2015-2016 - Situation épidémiologique au 30 juin 2016. Bulletin Epidémiologique.

Leinonen I., Williams A.G., Wiseman J., Guy J., Kyriazakis I., 2012a. Predicting the environmental impacts of chicken systems in the United Kingdom through a life cycle assessment: broiler production systems. Poult. Sci., 91, 8-25.

Leinonen I., Williams A.G., Wiseman J., Guy J., Kyriazakis I., 2012b. Predicting the environmental impacts of chicken systems in the United Kingdom through a life cycle assessment : egg production systems. Poult. Sci., 91, 26-40.

Litt J., 2015. Programme RENAPALM: gestiontechnico-économique des éleveurs et gaveurs de palmipèdes à foie gras. Résultats 2014, 66p
Maloux M., 1995. Dictionnaire des proverbes, sentences et maximes, Ed. Larousse, 628pp.

Miyake F.A., Takaesu Y., Kweon H. 2004. Identifying the image of a healing landscape A descriptive study. ISHS Acta Horticulturae 639: $26^{\text {th }}$ Int. Horticult. Congr. : Expanding Roles for Horticulture in Improving Human Well-Being and Life Quality.

Nair P.K.R., 1993. An Introduction to Agroforestry. Kluwer Academic Publishers and ICRAF, 496p

Pothin A., Meda B. Fortun-Lamothe L., 2017. Évaluer la compétition entre alimentation humaine et animale. 12 èmes Journ. Rech. Avicole et Palmipèdes à Foie Gras.

Puillet L., Martin O., Méda B., Garcia-Launay F., 2016. ArchiMod: a metamodel of farming systems functioning to address future livestock challenges. In Proc. 67th Ann. Meet. Europ. Fed. Anim. Sci., (EAAP), Belfast, U.K.

Prunet J.P., 2003. Fiche technico-économique "Verger type traditionnel" de la Station expérimentale de Creysse download at http:/ www.noixsudouest.fr/imgmaj/25/fiche-technico-economique-verger-traditionnel.pdf.

Regel D., 2014. Le merveilleux voyage de Nils Holgersson au pays des oies. DVD.

Rouvier R., 1992. L'amélioration génétique en France : le contexte et les acteurs - Les Palmipèdes. In : Numéro Hors-série, Éléments de génétique quantitative et application aux populations animales. Bibé B., Bonaïti B., Elsen
J.M., Guérin G., Mallard J., Minvielle F., de Mondini L., Mulsant P., De Rochambeau H., Farce M.H. (Eds). INRA Prod. Anim., 5, 39-43.

Ryschawy J., Tichit M., Bertrand S., Allaire G., Plantureux S., Aznar O., Perrot C., Guinot C., Josien E., Lasseur J., Aubert C., Tchakerian E., Disenhaus C., 2015. Comment évaluer les services rendus par l'élevage ? Une première approche méthodologique sur le cas de la France. INRA Prod. Anim., 28, 23-38.

Sauveur B., Rousselot-Pailley D., Larrue P., 1988. Alimentation énergétique de l'oie reproductrice. INRA Prod. Anim., 1, 209-214.

Sellier N., Rousselot-Pailley D., De Reviers M., 1995. L'insémination artificielle chez l'oie grise des Landes : bilan et perspectives. INRA Prod. Anim., 8, 127-133.

Smith J., Pearce B.D., Wolfe M.S., 2013. Reconciling productivity with protection of the environment: Is temperate agroforestry the answer? Renew. Agric. Food Sys., 28, 80-92. doi: $10.1017 / \mathrm{S} 1742170511000585$.

Steinfeld H., Gerber P., Wassenaar T., Castel V., Rosales M., de Haan C., 2006. In: Livestock's Long Shadow Environmental issues and options. Rome, Italy: FAO, 390 p. ftp://ftp.fao.org/docrep/ fao/010/a0701e/a0701e.pdf.

de Vries M., de Boer I.J.M., 2010. Comparing environmental impacts for livestock products: a review of life cycle assessments. Livest. Sci. 128, 1-11.

\section{Résumé}

L'élevage rend de nombreux services dans les territoires dans lesquels il est implanté et il est intéressant de pouvoir les appréhender dans leur globalité. L'objectif de cette étude est de réaliser une qualification du bouquet de services rendus par les systèmes de production de foie gras agroforestiers en prenant comme exemple l'association oies / noyers en Périgord. Le cadre conceptuel utilisé a été adapté de Ryschawy et al (2015). Les données proviennent 1) de mesures biologiques (croissance des arbres ou production de fruit, comportement des animaux, biodiversité) ou pédologiques (fertilité des sols) réalisées sur des oies adultes élevées sur des parcours contenant des noyers âgés de 7 à 11 ans et 2) des données de la littérature pour les autres dimensions. Notre analyse montre que l'élevage des oies pour la production de foie gras sur des parcours implantés de noyers est à bénéfices réciproques puisque l'ombre apportée par les noyers est très appréciée des animaux, et que les déjections des oies fertilisent les arbres ce qui augmente leur croissance et leur production de fruits sans altérer leur qualité sanitaire. Cette association rend des services $i$ ) d'approvisionnement par la fourniture en quantité relative modérée de foie gras, de viande d'oie, de bois de noyers, de noix et de coproduits (duvets et graisse d'oie, coques et brou de noix); ii) de préservation de la qualité environnementale en valorisants des coproduits dans l'alimentation des animaux $(77$ t/an) et en préservant la fertilité du sol ; iii) patrimoniaux et de qualité de vie par le biais de produits labellisés et à haute valeur ajoutée (AOC Noix du Périgord, marque Oie du Périgord) et le maintien du patrimoine culturel et gastronomique; et $i v$ ) de vitalité territoriale par la création d'emplois et la contribution à l'agrotourisme. Il a aussi des externalités négatives (impacts environnementaux, acceptabilité sociale du gavage et risques sanitaires). Ce travail permet de montrer que les bénéfices de l'association oies/noyers sont beaucoup plus larges que les intérêts techniques et économiques qui les ont justifiés.

\section{Abstract}

\section{Multiple services provided by agroforestry fatty liver production systems : the example of the geese/walnut association in Périgord}

Livestock provides many services in the territories in which it is established and it is interesting to be able to perceive them in their entirety. The objective of this study was to qualify the multiple contributions of agroforestry fatty liver production systems, taking as an example the association of geese/walnuts in Périgord. The conceptual framework used was that proposed by Ryschawy et al (2015). The data came from 1) biological measurements (tree growth or fruit production, animal behavior, biodiversity) or soil data (soil fertility) carried out on adult geese raised on outdoor areas of walnut trees aged between 7 and 11 years and 2) data from the literature, for the other dimensions. Our analysis shows that the breeding of geese for foie gras production on outdoor walnut areas is of mutual benefit since the shade provided by walnut trees is very appreciated by animals, while the droppings of the geese fertilize the trees, which increases their growth and their fruit production without altering their sanitary quality. This association provides services by $i$ ) of suppling the production of relative moderate amounts of foie gras, goose meat, walnut wood, walnuts and co-products (goose down and fat, walnut stain and shell); ii) preserving environmental quality by upgrading co-products in animal feed (77 t/year) and 
preserving soil fertility; iii) improving heritage and life quality through high added value products (AOC Noix du Périgord, goose of Périgord brand) and preserving gastronomic and cultural heritage iv) favoring territorial vitality through employment and a contribution to agro-tourism. It also has negative aspects (environmental impacts, social acceptability of gavage and health risks). This work shows that the benefits of the goose/walnut association are much broader than the technical and economic interests that justified them.

BIJJA M., ARROYO J., LAVIGNE F., DUBOIS J.-P., FORTUN-LAMOTHE L., 2017. Les services rendus par les systèmes de production de foie gras agroforestiers : l'exemple de l'association entre oies et noyers en Périgord. INRA Prod. Anim., 30, 241-254. 
